\title{
Installation des crèches dans un emplacement public : des critères flous
}

Hélène Pauliat

\section{OpenEdition}

1 Journals

Édition électronique

URL : https://journals.openedition.org/rdr/692

DOI : $10.4000 /$ rdr.692

ISSN : 2534-7462

Éditeur

Presses universitaires de Strasbourg

\section{Édition imprimée}

Date de publication : 31 octobre 2017

Pagination : 67-82

ISBN : 978-2-86820-974-0

ISSN : 2493-8637

\section{Référence électronique}

Hélène Pauliat, "Installation des crèches dans un emplacement public : des critères flous », Revue du droit des religions [En ligne], 4 | 2017, mis en ligne le 15 janvier 2020, consulté le 23 mai 2022. URL: http://journals.openedition.org/rdr/692 ; DOI : https://doi.org/10.4000/rdr.692

\section{(a) $(1)(9$}

La revue du droit des religions est mise à disposition selon les termes de la Creative Commons Attribution - Pas d'Utilisation Commerciale 4.0 International - CC BY-NC 4.0. 


\section{INSTALLATION DES CRÈCHES DANS UN EMPLACEMENT PUBLIC : DES CRITÈRES FLOUS}

\section{Hélène PAULIAT}

Université de Limoges, Observatoire des mutations institutionnelles et juridiques (OMIJ)

\section{RÉSUMÉ}

À quelles conditions l'implantation d'une crèche dans un bâtiment public est-elle légale? Une telle question ne s'était guère posée jusqu'à ces dernières années, mais le contexte a profondément changé, du fait de la montée en puissance de revendications identitaires et de la volonté de certains élus locaux de manifester leurs convictions. Les décisions rendues par le Conseil d'État le 9 novembre 2016 apportent des éléments de réponse, mais ne mettent pas fin à l'ensemble des interrogations. En reconnaissant le principe de neutralité des personnes publiques, la haute instance précise l'interprétation de l'article 28 de la loi de 1905, en distinguant selon que la crèche est placée dans un bâtiment public ou dans un emplacement public. Les critères retenus ne seront pas toujours aisés à manier.

\section{ABSTRACT}

What conditions are required to display Christmas cribs in public buildings? Such a question hardly arose until recent years, in a context of growing identity claims and in which elected local officials want to express their beliefs. In its decisions of 9 November 2016, the Council of State provides some clues, but does not conclude the questioning. By recognizing the principle of neutrality of public persons, the High Court clarifies the interpretation of Article 28 of the 1905 Act, in distinguishing between cribs placed in a public building or in a public place. But the chosen criteria may not be easy to use. 
T 'on attendait une décision du Conseil d'État pour mettre fin aux dis_cordances des solutions rendues par les tribunaux administratifs et les cours administratives d'appel sur la question de savoir si l'installation de crèches dans des emplacements publics était légale ou non... Si la Haute juridiction administrative s'est effectivement prononcée, dans sa formation la plus solennelle, le 9 novembre 2016, les deux arrêts d'assemblée n'apportent pas toutes les clarifications souhaitées et ne vont probablement pas mettre un terme à tous les contentieux en ce domaine.

À l'origine des décisions du Conseil d'État, les requêtes de la Fédération départementale des libres penseurs de Seine-et-Marne et de la Fédération de la libre pensée de Vendée ${ }^{1}$, contestant, pour la première, l'installation d'une crèche de Noël dans l'enceinte de l'hôtel de ville de Melun, pour la seconde, l'installation d'une crèche de Noël dans les locaux de l'hôtel du département de la Vendée, à La Roche-sur-Yon. Dans les deux cas, les requérants souhaitaient que le juge administratif ordonne aux autorités publiques locales d'enlever ce symbole religieux des bâtiments publics, au nom de la neutralité et de la laïcité des personnes publiques. Le tribunal administratif de Melun avait, par un jugement du 22 décembre $2014^{2}$, rejeté la demande de la Fédération des libres penseurs de Seine-et-Marne, mais la cour administrative d'appel de Paris avait finalement fait droit à sa demande ${ }^{3}$, en estimant que « contrairement à ce qu'ont estimé les premiers juges, une crèche de Noël, dont l'objet est de représenter la naissance de Jésus, installée au moment où les chrétiens célèbrent cette naissance, doit être regardée comme ayant le caractère d'un emblème religieux [...] et non comme une simple décoration traditionnelle ", son installation est donc contraire aux dispositions de la loi de 1905 de séparation des Églises et de l'État, et au principe de neutralité des services publics. De son côté, le tribunal administratif de Nantes avait, par un jugement du 14 novembre $2014^{4}$, donné raison à la Fédération de la libre pensée de Vendée, mais la cour administrative d'appel de Nantes avait annulé ce jugement ${ }^{5}$, en estimant que la crèche « s'inscrit dans le cadre d'une tradition relative à la préparation de la fête familiale de Noël et ne revêt pas la nature d'un "signe ou emblème religieux" [...] ; alors même qu'elle ne se rattache pas à un particularisme local », elle ne méconnaît ni les dispositions de la loi de 1905 ni les principes de liberté de conscience et de neutralité du

1. CE, ass., 9 nov. 2016, $\mathrm{n}^{\circ} 395122$ et $\mathrm{n}^{\circ} 395223$.

2. TA Melun, 22 déc. 2014, $n^{\circ} 1300483$.

3. CAA Paris, 8 oct. 2015, n 15PA00814, concl. Romnicianu, BJCL 2015, p. 781.

4. TA Nantes, 14 nov. 2014, $\mathrm{n}^{\circ} 1211647$.

5. CAA Nantes, 13 oct. 2015, n 14NT03400, concl. GAuthier, BJCL 2015, p. 777. 
service public. Dans un contexte marqué par la méfiance à l'égard de certaines revendications religieuses qui apparaissent en contradiction totale avec les principes et valeurs de la République, cette «affaire des crèches » soulevait la question de la tolérance des pouvoirs publics à l'égard de l'implantation dans des emplacements publics de signes religieux ou susceptibles d'être ainsi qualifiés... L'imprécision des différents termes souligne à quel point la réponse à cette question semble délicate, les pratiques des communes ou des départements ayant jusqu'à présent fait office de droit applicable.

Le Conseil d'État était donc confronté à un problème redoutable : appliquer les textes de la République qui posent des règles en la matière, sans renier totalement un héritage judéo-chrétien qui fait partie intégrante de notre patrimoine, sans pour autant être constamment revendiqué ; conforter le vivre ensemble en ne heurtant aucune conscience, sans remettre en cause des traditions ancestrales... D'où la difficulté pour la haute instance à rendre une décision équilibrée et claire, au moment où tout signe peut être considéré comme véhiculant un message. Les juges du Palais-Royal ont ainsi rappelé l'interprétation logique de la loi de 1905, en particulier de son article 28 (1), avant de tenter d'appliquer cette interprétation au cas spécifique des crèches (2). Au regard des éléments fournis par les décisions, il apparaît que les critères présentés ne sont ni d'une grande clarté ni d'un maniement aisé pour les décideurs publics locaux.

\section{UNE INTERPRÉTATION LOGIQUE DE LA LOI DE 1905}

L'analyse de la loi du 9 décembre 1905 de séparation des Églises et de l'État conduit le juge à dégager un principe général d'interdiction (1.1) et des exceptions à ce principe (1.2), ce qui apparaît dans une totale continuité avec les solutions rendues jusqu'alors.

\subsection{UN PRINCIPE GÉNÉRAL D'INTERDICTION D'APPOSER UN SIGNE RELIGIEUX SUR UN MONUMENT OU UN EMPLACEMENT PUBLIC}

Selon les dispositions constitutionnelles en vigueur, la France est une République laïque; elle assure l'égalité devant la loi de tous les citoyens sans distinction de race, d'origine ou de religion; elle respecte toutes les croyances. Ce principe, posé par l'article $1^{\text {er }}$ de notre texte fondamental, est largement décliné dans ses différents aspects ; il n'est, pour s'en convaincre, 
que de se reporter aux derniers textes publiés, qui souhaitent renforcer l'effectivité du principe d'égalité en améliorant la lutte contre les discriminations, par exemple les modifications apportées par la loi du 18 novembre 2016 de modernisation de la justice du XxI $^{e}$ siècle à la loi du 27 mai 2008 portant diverses dispositions d'adaptation au droit communautaire dans le domaine de la lutte contre les discriminations ${ }^{6}$. Le législateur a par ailleurs cherché à protéger les fonctionnaires et agents publics de toute mesure qui pourrait être prise en leur défaveur fondée sur leurs opinions religieuses ${ }^{7}$.

La loi du 9 décembre 1905 impose, pour les personnes publiques, un certain nombre d'obligations. Considérée comme la « clé de voûte de la laïcité ${ }^{8} »$, elle s'efforce de définir le régime juridique des relations entre l'État et les cultes. Comme le rappelle le Conseil d'État dans une présentation synthétique de l'esprit de la $\operatorname{loi}^{9}$, les personnes publiques doivent assurer à tous la liberté de conscience et garantir le libre exercice des cultes, ce que traduit l'article $1^{\text {er }}$ de la loi portant séparation des Églises et de l'État. Cette obligation positive a été concrètement appliquée devant les juridictions administratives, dans plusieurs décisions d'importance ${ }^{10}$. Assurer la liberté

6. La loi, modifiée en dernier lieu par la loi du 28 février 2017, procède à une reformulation de l'interdiction de discriminer une personne en fonction de "son origine, de son sexe, de sa situation de famille, de sa grossesse, de son apparence physique, de la particulière vulnérabilité résultant de sa situation économique, apparente ou connue de son auteur, de son patronyme, de son lieu de résidence ou de sa domiciliation bancaire, de son état de santé, de sa perte d'autonomie, de son handicap, de ses caractéristiques génétiques, de ses mœurs, de son orientation sexuelle, de son identité de genre, de son âge, de ses opinions politiques, de ses activités syndicales, de sa capacité à s'exprimer dans une langue autre que le français, de son appartenance ou de sa non-appartenance, vraie ou supposée, à une ethnie, une nation, une prétendue race ou une religion déterminée * (art. 86 de la loi du 18 novembre 2016 modifiant l'article $1^{\text {er }}$ de la loi du 27 mai 2008).

7. Voir l'article 6 de la loi du 13 juillet 1983 modifiée relative aux droits et libertés des fonctionnaires : "Aucune distinction, directe ou indirecte, ne peut être faite entre les fonctionnaires en raison de leurs opinions politiques, syndicales, philosophiques ou religieuses, de leur origine, de leur orientation sexuelle ou identité de genre, de leur âge, de leur patronyme, de leur situation de famille, de leur état de santé, de leur apparence physique, de leur handicap ou de leur appartenance ou de leur non-appartenance, vraie ou supposée, à une ethnie ou une race. "

8. France. Conseil d'État, Un siècle de laïcité, Paris, La Documentation française, 2004, p. 258.

9. CE, ass., 9 nov. 2016, n 395122, Fédération départementale des libres penseurs de Seineet-Marne, cons. 3.

10. Les dispositions de la loi du 9 décembre 1905 n'interdisent pas à une " commune, en tenant compte des nécessités qu'elles mentionnent, d'autoriser, dans le respect du principe de neutralité à l'égard des cultes et du principe d'égalité, l'utilisation d'un local qui lui appartient pour l'exercice d'un culte par une association, dès lors que les conditions financières de cette autorisation excluent toute libéralité et, par suite, toute aide à un culte; qu'une commune ne peut rejeter une demande d'utilisation d'un tel local au seul 
de conscience implique que les personnes publiques mettent en place les personnels nécessaires pour que certains usagers soient en mesure d'exercer leur culte, dans les prisons ou dans les hôpitaux par exemple, au risque de voir leur responsabilité engagée. Mais, en complément de ces exigences, la loi de 1905 impose aux personnes publiques de veiller à la neutralité des agents publics et des services publics à l'égard des cultes, en particulier en n'en reconnaissant et en n'en subventionnant aucun, conformément à l'article 2 du texte. Le juge administratif veille au respect de cette interdiction de subvention, en recherchant, dans chaque litige qui lui est soumis, si la somme versée par une collectivité publique peut ou risque d'être utilisée pour l'exercice d'un culte ${ }^{11}$. La neutralité des agents publics trouve désormais une traduction concrète dans le statut général des fonctionnaires, modifié par la loi du 20 avril $2016^{12}$, alors même que le juge leur avait depuis longtemps imposé une telle obligation ${ }^{13}$. Cette neutralité des personnels se fonde elle-même sur la neutralité des services publics qui, elle, a valeur constitutionnelle ${ }^{14}$.

Ces différents principes sont mis en œuvre par l'article 28 de la loi de 1905. Cette disposition, qui interdit, sauf exceptions particulières, d'apposer pour l'avenir un signe ou un emblème religieux sur des monuments ou emplacements publics, a pour objet « d'assurer la neutralité des personnes publiques à l'égard des cultes ${ }^{15} »$; elle s'oppose donc à l'installation par celles-ci, dans un emplacement public, d'un signe ou emblème manifestant la reconnaissance d'un culte ou marquant une préférence religieuse. Le

motif que cette demande lui est adressée par une association dans le but d'exercer un culte» (CE, ass., 19 juill. 2011, $\mathrm{n}^{\circ}$ 313518, Commune de Montpellier).

11. CE, ass., 19 juill. 2011, n 308544, Commune de Trélazé, le juge précisant que lorsqu'un orgue, acheté par une collectivité publique, est installé dans une église, son utilisation doit être faite prioritairement pour un intérêt public et, s'il est utilisé par le prêtre, faire l'objet d'une convention précisant les modalités financières.

12. Article 25 de la loi du 20 avril 2016 relative à la déontologie et aux droits et obligations des fonctionnaires : «Le fonctionnaire exerce ses fonctions dans le respect du principe de laïcité. À ce titre, il s'abstient notamment de manifester, dans l'exercice de ses fonctions, ses opinions religieuses. Le fonctionnaire traite de façon égale toutes les personnes et respecte leur liberté de conscience et leur dignité. »

13. France. Conseil d'État, Un siècle de laïcité, op. cit., p. 272 et s.

14. Même si la décision du Conseil constitutionnel que l'on cite généralement n'est pas aussi claire dans son affirmation (18 sept. 1986, n 86-217 DC, Loi relative à la liberté de communication), cons. 15 : les cahiers des charges qui imposent certaines exigences aux sociétés du secteur public de l'audiovisuel « doivent nécessairement se conformer aux principes fondamentaux du service public et notamment au principe d'égalité et à son corollaire le principe de neutralité du service».

15. CE, ass., 9 nov. 2016, nº 395122. 
principe d'interdiction est alors clairement formulé : le signe ou emblème religieux est identifié en ce qu'il marque une préférence religieuse ou manifeste la reconnaissance d'un culte; en revanche, le monument public ou l'emplacement public n'est guère défini. Il faut se reporter aux travaux préparatoires de la loi et aux discussions qui ont présidé à son vote, rappelés par le rapporteur public Aurélie Bretonneau ${ }^{16}$ : les emplacements publics sont des propriétés de l'État, du département, de la commune (il faudrait désormais ajouter la région, les établissements publics, les établissements publics de coopération intercommunale...), mais aussi les rues, les places publiques, les édifices publics autres que les églises et les musées. Les emplacements publics englobent donc « tous les monuments ou terrains relevant du domaine public ${ }^{17}$ » des personnes publiques. La prohibition s'avère logique au regard de l'interprétation de la loi de 1905 et de la nécessaire neutralité publique, personnels comme services.

La loi ménage cependant certaines exceptions, que le juge administratif ne reprend que pour partie.

\subsection{DES EXCEPTIONS LIMITÉES À L'INTERDICTION GÉNÉRALE MÉNAGEANT UNE FAIBLE LATITUDE AUX PERSONNES PUBLIQUES}

Si la loi de 1905 pose un principe d'interdiction d'apposer des signes religieux sur des monuments publics, elle ne le met en œuvre que pour l'avenir, d'une part, et elle exclut certains lieux ou manifestations de cette interdiction.

La loi de 1905 n'interdit l'apposition de signes religieux que pour l'avenir, ce qui visait à préserver les signes et emblèmes religieux existants à la date d'entrée en vigueur de la loi ; une application immédiate aurait conduit à défigurer certains monuments ou emplacements publics et, par voie de conséquence, à susciter des heurts supplémentaires entre partisans d'une laïcité militante et chrétiens convaincus... Cette entrée en vigueur tempère donc l'immédiateté de l'interdiction.

Mais le texte tolère des exceptions : il est donc possible d'apposer des signes religieux dans les édifices servant au culte, sur les terrains de sépulture dans les cimetières, sur des monuments funéraires et dans des musées ou lors d'expositions. Dans l'arrêt du 9 novembre 2016, le Conseil d'État ne reprend qu'une partie de ces exceptions : les personnes publiques ont la

16. Conclusions sur les deux arrêts d'assemblée du 9 novembre 2016.

17. Selon la formule d'Aurélie Bretonneau. 
possibilité d'apposer des signes religieux «dans un emplacement public à titre d'exposition ». La formule retenue n'apparaît pas très satisfaisante, elle impose en effet une définition juridique, absente de la décision. L'exposition peut-elle se faire dans n'importe quel emplacement public? L'exposition suppose-t-elle un caractère temporaire, la permanence empêchant l'usage de ce terme? Pour le rapporteur public, « la présentation de pièces religieuses ou culturelles ne saurait troubler l'ordre public et la liberté de conscience dès lors que la vocation artistique et pédagogique de l'exposition fait écran entre la charge religieuse des objets et la charge symbolique que véhicule leur présentation par une personne publique ». Les autres exceptions ne sont pas explicitées : autant elles paraissent évidentes pour les édifices du culte, qui postulent quasi naturellement l'apposition de signes religieux, autant elles le sont moins pour les terrains de sépulture dans les cimetières, qui sont des lieux publics civils, dans lesquels donc toute marque d'une confession est prohibée dans les parties communes; il est utile de rappeler que seules les tombes peuvent éventuellement faire apparaître des signes particuliers mettant en évidence la religion de la personne décédée ${ }^{18}$. Les monuments funéraires peuvent recevoir des signes religieux, mais encore faudrait-il préciser que les monuments aux morts, par exemple, demeurent dans le champ d'application de l'interdiction, sauf s'ils sont autrement qualifiés ${ }^{19}$.

Ces éléments ayant été précisés par le Conseil d'État, il semblait que la solution pouvait alors être simple; si elle ne l'est pas, c'est parce que la crèche de Noël revêt une pluralité de significations.

18. V. par ex. la circulaire NOR INTA0800038C du ministre de l'Intérieur du 19 février 2008 relative à la police des lieux de sépulture (aménagement des cimetières et regroupements confessionnels des sépultures). L'existence de carrés confessionnels est interdite, les circulaires ayant cependant clairement encouragé les maires, à l'encontre de l'esprit et de la lettre de la loi de 1905, à aménager « en fonction des demandes, des espaces regroupant les défunts de même confession, en prenant soin de respecter le principe de neutralité des parties communes du cimetière ainsi que le principe de liberté de croyance individuelle».

19. V. CAA Lyon, 16 mars 2010, n 07LY02583 : « le monument élevé à la mémoire des morts de la guerre de 1914-1918, initialement sur un emplacement situé devant l'église de ladite commune, doit être considéré comme un monument funéraire au sens des dispositions précitées de l'article 28 de la loi du 9 décembre 1905, nonobstant la circonstance qu'il n'a pas été érigé dans un cimetière; que, par suite, l'apposition de signes ou emblèmes religieux sur ce monument n'était pas interdite par cet article. » 


\section{UNE APPLICATION DIFFICILE AUX CRÈCHES DE NOËL}

La crèche de Noël étant une représentation de la Nativité, donc d'une scène religieuse, il était possible d'estimer que l'on devait appliquer à cet emblème religieux les principes rappelés par la haute instance. Toute crèche serait alors interdite dans les emplacements publics, sauf si elle était en lien avec les exceptions prévues par la loi de 1905. Prenant acte de l'évolution de la société, le Conseil d'État se fonde sur la diversité des significations d'une crèche pour établir des critères permettant de considérer comme étant légale son installation dans un emplacement public (2.1), dissociant alors le contexte de l'installation du lieu de l'installation, sans s'en tenir à l'objet même ou au signe ${ }^{20}$. Le maniement de ces critères demeure délicat et suscite donc l'interrogation quant à leur pertinence (2.2).

\subsection{LA PLURALITÉ DES CRITÈRES}

La difficulté fondamentale à laquelle était confronté le Conseil d'État était de déterminer si la crèche de Noël entrait ou non dans le champ d'application de l'article 28 de la loi du 9 décembre 1905 : constituait-elle un signe ou emblème religieux ${ }^{21}$ ? C'est sur cette question que s'étaient opposés les juges du fond, certains considérant qu'elle était par elle-même un symbole religieux, relevant donc de l'interdiction générale posée par la loi de Séparation, les autres estimant au contraire qu'elle avait perdu toute signification religieuse, constituant seulement une décoration, parmi d'autres, des fêtes de fin d'année. La haute instance ne tranche pas ce débat, il eût été d'ailleurs surprenant qu'elle le fît. Elle se contente de constater la «pluralité de significations » de la crèche de Noël désormais, scène faisant partie de l'iconographie chrétienne, certes, mais élément indissociable des décorations et illustrations des fêtes de fin d'année, sans signification religieuse particulière ${ }^{22}$. Cette réponse double du Conseil d'État implique alors que la crèche de Noël relève, pour partie seulement, de l'interdiction posée, ou si l'on préfère, pour sa partie ou sa

20. Dutheillet de Lamothe V., Odinet G., « La crèche entre dans les tables », AJDA 2016, p. 2375.

21. Chifflot N., « L'affaire des crèches de Noël devant le Conseil d'État, rendre à César ce qui est à César», JCP A 2016, 2309.

22. Le rapporteur public Aurélie Bretonneau soulignait, après un panorama historique des crèches, « une déconnection progressive de la crèche de son substrat religieux ». 
signification religieuse ; elle apparaît comme un «objet mixte ${ }^{23} »$. Le juge doit ainsi dégager des critères permettant de déterminer, dans chaque cas, si la crèche relève davantage de la signification religieuse, donc du cultuel, ou davantage de la signification festive ou culturelle.

Selon le juge, et compte tenu de ces différents sens, «l'installation d'une crèche de Noël, à titre temporaire, à l'initiative d'une personne publique, dans un emplacement public, n'est légalement possible que lorsqu'elle présente un caractère culturel, artistique ou festif, sans exprimer la reconnaissance d'un culte ou marquer une préférence religieuse ». L'objectif est donc bien de privilégier le culturel sur le cultuel. Au regard des principes dégagés dès le début de la décision, le Conseil d'État en déduit que l'installation d'une crèche symbolisant l'iconographie chrétienne est interdite; elle ne devient légale que si l'on démontre qu'elle répond à un intérêt ou à une démarche festive, culturelle ou artistique. Le caractère festif est lié aux fêtes de fin d'année et n'était guère envisagé par la loi de 1905 ; en revanche, le caractère culturel renvoie indéniablement à la présence de cette représentation dans les musées ou à titre d'expositions. La référence au caractère «temporaire » de la mise en évidence se comprend alors, mais le rapporteur public insistait sur le fait que «le caractère limité dans le temps ne suffit pas à lui seul à lever l'interdit » d'apposition du signe religieux. Dès lors que la crèche apparaît comme étant une pièce de collection, ou une œuvre présentant un intérêt historique majeur, elle peut être considérée comme une "exposition » au sens de la loi de 1905 et entrer pleinement dans le champ des exceptions prévues ${ }^{24}$.

Mais comment procéder pour déterminer si la crèche de Noël répond plutôt au culturel ou au cultuel ? Quel est le sens principal de l'objet, quel est son sens accessoire? Selon la haute instance, il faut tenir compte «non seulement du contexte qui doit être dépourvu de tout élément de prosélytisme, des conditions particulières de cette installation, de l'existence ou de l'absence d'usages locaux, mais aussi du lieu de cette installation ». Le juge divise donc entre contexte et lieu de l'installation de la crèche.

23. Selon la formule retenue par le rapporteur public. V. également MAUS D., «Une crèche est-elle un objet cultuel ou culturel ? », D. 2016, p. 2456 ; Dieuleveult A. DE, « Noël au risque de la laïcité », AJDA 2015, p. 2390.

24. V. par ex. une décision du tribunal administratif de Montpellier du 16 juillet 2015 ( ${ }^{\circ}$ 1405625) : «l'installation de la crèche dans l'hôtel de ville de Béziers a constamment été présentée comme une exposition s'inscrivant dans le cadre d'animations culturelles organisées à l'occasion des fêtes de Noël [...] sans qu'aucun élément du dossier ne vienne révéler une intention différente et/ou la manifestation d'une préférence pour les personnes de confession chrétienne. » 
S'agissant du contexte, l'on comprend qu'il faille éviter les références religieuses prononcées, qui permettraient de voir du prosélytisme, comme des croix autour de la représentation, ou encore une bible, voire des discours présidant à son inauguration, ou des prières autour de l'exposition... L'existence ou l'absence d'usages locaux se comprend également : si une crèche est installée dans un emplacement public depuis des dizaines d'années sans que cela pose problème, l'on pourrait admettre que l'usage s'en est imposé ; mais il est quand même difficile d'avaliser le fait que la pratique et les traditions l'emportent sur le principe d'interdiction posé par la loi de 1905... À l'inverse, et comme cela a pu être le cas dans certaines mairies, l'installation d'une crèche dans un emplacement public, uniquement liée à un changement de majorité et à l'arrivée d'un maire ou d'un exécutif voulant souligner la primauté de la religion chrétienne et des représentations catholiques sur toute autre, orienterait le juge vers le caractère cultuel de la crèche ${ }^{25}$. Bien entendu, l'époque à laquelle la crèche est installée est un indice important, même si l'on a du mal à imaginer qu'une collectivité prenne la décision d'installer une représentation de la Nativité en dehors des fêtes de fin d'année! Mais qu'en est-il des conditions particulières de l'installation? Le juge n'en dit mot. Si l'on se réfère aux conclusions du rapporteur public Éric Gauthier devant la cour administrative d'appel de Nantes ${ }^{26}$, il serait important d'établir si la crèche atteste d'une volonté de célébration religieuse. Plus précisément, l'emplacement de la crèche serait un indice du caractère cultuel ou non : est-elle placée dans un endroit discret ou au contraire à la vue de tous, est-elle de taille modeste ou au contraire imposante, est-elle la seule représentation ou au contraire s'accompagne-t-elle d'autres décors rappelant les fêtes de Noël ${ }^{27} \ldots$ ? Il est un peu dommage que le Conseil d'État n'ait pas souhaité se montrer plus précis sur cet aspect à prendre en compte.

S'agissant du lieu de l'installation de la crèche, le juge estime que « la situation est différente » selon qu'il s'agit d'un bâtiment public ou d'un emplacement public ${ }^{28}$. On peut être surpris de cette distinction, absente de la loi du 9 décembre 1905. L'emplacement public y était appréhendé

25. C'est ce que soulignait le rapporteur public : «l'apparition soudaine d'une crèche dans l'espace public, alors que la municipalité ne s'était jamais préoccupée de célébrer Noël et ne se livre à aucune initiative en matière de décoration, dans un contexte local de crispation sur la question religieuse, pourra être l'indice d'une revendication. »

26. CAA Nantes, 13 oct. 2015, précit.

27. V. Pauliat H., "Crèches et bâtiments publics, la discorde », JCP A 2015, act. 1002.

28. Verpeaux M., « Crèches dans les établissements publics : non, mais », JCP G 2016, p. 2433. 
de manière générique, comme un bien relevant du domaine public d'une personne publique ${ }^{29}$. Selon le juge, si le lieu choisi pour la crèche est l'enceinte d'un bâtiment public, siège d'une collectivité publique ou d'un service public, l'installation par la personne publique de cette représentation est contraire aux exigences découlant du principe de neutralité des personnes publiques, sauf si elle parvient à démontrer l'existence de circonstances particulières permettant de reconnaître à la crèche un caractère culturel, artistique ou festif. Compte tenu de la rédaction de l'arrêt, le principe posé ici par le Conseil d'État pose problème. Selon le considérant 5, il est indispensable de distinguer le caractère culturel, artistique ou festif d'une crèche, d'un caractère purement cultuel. Pour opérer cette distinction, le même considérant distingue des éléments liés au contexte et des éléments liés au lieu d'installation, ce qui entraîne une nouvelle distinction bâtiment public ou emplacement public; mais dans le cadre du bâtiment public, le juge revient sur le critère initial culturel/cultuel, ce qui ne rend pas la démarche lisible. In fine, pour certains bâtiments publics, ceux qui sont le siège d'une collectivité publique ou d'un service public, le principe est l'interdiction de l'installation d'une crèche sauf si le caractère culturel est principal ${ }^{30}$, voire exclusif. La crèche ne doit pas marquer une préférence religieuse, c'est sur cette approche que se fonde le Conseil d'État pour respecter l'article 28 de la loi de 1905. Mais, dans les autres emplacements publics, et donc dans l'enceinte des bâtiments publics qui n'abritent ni une collectivité, ni un service public, le principe posé est inverse : l'installation d'une crèche est possible, le juge semblant même estimer qu'il existerait une sorte de présomption de caractère festif de la représentation en question, liée à la période (fêtes de fin d'année), au lieu (voie publique), au contexte (décorations en lien avec la période), sauf à démontrer que la crèche manifeste une revendication religieuse, faisant primer le caractère cultuel sur le caractère culturel. En clair, si la crèche se situe dans un bâtiment public au sens de

29. Les chroniqueurs de l'AJDA s'en étonnaient également (Dutheillet de LAmothe V., Odinet G., précit.) : "la distinction entre les bâtiments publics qui sont le siège d'une collectivité publique ou d'un service public et les autres emplacements publics n'est pas sans étonner. Outre que la loi traite uniformément les emplacements publics, la frontière aussi nette ainsi créée peine à convaincre, qui unit le hall de gare et la salle du conseil municipal mais sépare, dans une approche centimétrée de la légalité, le porche de l'hôtel de ville de son parvis.»

30. C'est de cette manière que s'est prononcé le tribunal administratif de Lille, dans la première application par un juge du fond des principes posés par les arrêts du Conseil d'État du 9 novembre 2016 (TA Lille, 30 nov. 2016, n 1509979), à propos de l'installation d'une crèche dans le hall de l'hôtel de ville d'Hénin-Beaumont; une telle installation est en principe prohibée, sauf circonstances particulières. 
l'arrêt, la présomption est qu'elle a un caractère cultuel, donc prohibé, sauf à prouver que son caractère culturel est principal; si la crèche se situe dans un autre emplacement public, la présomption est que le caractère culturel et festif prime, sauf à prouver que son caractère cultuel et revendicatif prime... Voilà une distinction qui ne sera probablement pas facile à manier pour nos représentants locaux!

L'application des critères par le Conseil d'État n'est pas aisée non plus. Le juge s'est en effet prononcé sur le fond dans l'affaire de la crèche implantée « dans une alcôve située sous le porche reliant la cour d'honneur au jardin de l'hôtel de ville de Melun ». Il retient que le lieu d'installation de la crèche est l'enceinte d'un bâtiment public, siège d'une collectivité publique. Cela implique que, en principe, l'installation est interdite, sauf à prouver le caractère festif, culturel ou artistique. Or, selon le Conseil, cette installation « n'était accompagnée d'aucun autre élément marquant son inscription dans un environnement culturel, artistique ou festif »; les « circonstances particulières » évoquées plus haut par le juge sont ainsi absentes ${ }^{31}$. Le contexte est très rapidement écarté, aucun usage local ne pouvant servir de justification, seule la période (fêtes de la fin d'année 2012) pouvant être un élément favorable au caractère festif ; l'installation méconnaît alors l'article 28 de la loi de 1905. À l'analyse de la démarche du Conseil d'État, il semble donc qu'il faille conclure au fait que les critères ne sont pas équivalents; le juge s'interroge d'abord sur le lieu de l'installation, et en fonction de ce lieu, utilise les critères présentés initialement, en partant d'une présomption ou tout au moins d'un principe.

La décision rendue soulève une dernière interrogation, liée à la référence $\mathrm{au}$ « principe de neutralité des personnes publiques ». S'agit-il d'un raccourci renvoyant à la neutralité des personnes publiques à l'égard des cultes, d'un principe englobant le principe de neutralité des services publics, des emplacements publics et des agents publics, ou le juge a-t-il voulu consacrer un nouveau principe, plus large que celui de la neutralité de l'État?

La lecture de l'arrêt amène à s'interroger sur la pertinence des critères retenus.

31. Alors même que le rapporteur public estimait que, « dans la majeure partie des cas, l'installation d'une crèche dans l'espace public ne posera pas de difficulté dès lors qu'elle s'insèrera sans distinction ou mise en valeur particulière, par la mise en scène ou le discours, dans un ensemble de décorations festives $»$. 


\subsection{LA PERTINENCE DES CRITÈRES}

Les critères retenus par le Conseil d'État n'apparaissent pas totalement lisibles. Ils ne contribuent pas complètement à la cohérence des solutions rendues par le juge, qui avait déjà, à maintes reprises, pourtant, interprété de manière libérale la loi de 1905. Il n'est peut-être alors pas inutile, en se fondant sur ce «principe de neutralité des personnes publiques », de s'interroger sur d'autres critères, qui fondaient les solutions en matière de neutralité des agents publics et en matière d'interdiction de subventions aux cultes.

Les solutions rendues par le Conseil d'État sur le fondement de l'article 2 de la loi du 9 décembre 1905, selon lequel la République ne subventionne aucun culte, se sont fondées sur l'existence ou non d'un intérêt public local pour mettre en évidence le caractère non cultuel du bien meuble ou immeuble en cause, et sur la signature d'une convention, permettant de s'assurer que, si le bien pouvait servir à l'exercice d'un culte, ce n'était que moyennant une rémunération excluant toute subvention déguisée. Ce sont des critères plus difficiles à exploiter qui ont été retenus par le Conseil d'État dans les arrêts du 9 novembre 2016; ils ne se rattachent pas non plus à la jurisprudence relative aux ports de signes religieux par les agents publics. La jurisprudence aurait pourtant, semble-t-il, gagné en cohérence.

Ainsi, l'installation de la crèche répond-elle à un intérêt public local, qui pourrait être de nature artistique, culturelle, ethnographique, ou encore, pourquoi pas, touristique, du fait de ses particularités qui seraient susceptibles d'accueillir de nombreux touristes pour la visiter ${ }^{32}$ ? Dans ce dernier cas, elle serait probablement accompagnée d'un environnement spécifique, de nature ornementale. Cet intérêt public local pourrait également être de nature historique, prenant ainsi en compte les traditions associées à cette représentation. Une fois ce premier élément identifié, justifiant de ne pas reconnaître à cette crèche un caractère purement cultuel, un deuxième élément pourrait faire l'objet d'une appréciation : la nature de l'implantation. Le lieu dans lequel est installée la crèche constitue-t-il un emplacement public, sans qu'il y ait lieu de distinguer, de manière quelque peu artificielle, entre emplacements publics et bâtiments publics, sièges d'une collectivité ou d'un service public? Ce lieu est-il un simple lieu de passage du public ou au contraire un lieu

32. Le TA de Lille a tenté de raisonner de cette manière, dans le jugement précité : la présence de la crèche dans le hall de l'hôtel de ville n'avait pas de lien caractérisé avec la tenue simultanée du marché de Noël dans la commune, qui a été déployé à l'extérieur du bâtiment sans proximité directe avec l'hôtel de ville et il n'existe pas de tradition locale préexistante en ce sens à Hénin-Beaumont. 
dans lequel le public est reçu longuement, ou encore un lieu dans lequel les élus délibèrent? Si la réponse est affirmative, les caractéristiques de la crèche sont-elles de nature à la regarder comme une représentation revendiquant une préférence religieuse? Ainsi, par sa dimension, par exemple, par la manière dont elle est exposée, de manière ostensible ou discrète, par la manière même dont elle est présentée, la crèche peut-elle s'analyser ou non en un acte de prosélytisme? Enfin, l'emplacement retenu pour installer la crèche accueille-t-il, tout au long de l'année, d'autres représentations, d'autres expositions? Ce sont certains de ces éléments que le rapporteur public évoquait dans ses conclusions sur les décisions de novembre 2016, mais sans être totalement suivi par le Conseil d'État dans le raisonnement proposé.

De tels critères permettraient de relier les éléments requis par la jurisprudence pour assurer le respect du principe de neutralité des services publics et des bâtiments publics et ceux qui sont requis pour assurer le respect du principe de neutralité des agents publics. La jurisprudence serait ainsi lisible pour faire respecter, cette fois-ci, le principe de neutralité des personnes publiques, dans un souci de cohérence, et surtout de lisibilité pour les élus locaux.

Les décisions rendues en assemblée par le Conseil d'État ne vont probablement pas mettre fin aux contentieux relatifs à l'installation des crèches au sein d'emplacements publics ${ }^{33}$. Il sera indispensable d'affiner les critères permettant de reconnaître la légalité ou l'illégalité des décisions d'installation des crèches, tant les élus locaux auront des difficultés à manier les éléments actuels. La voie est tracée, il est utile de préciser la mise en œuvre du principe de neutralité des personnes publiques.

33. Il est d'ailleurs surprenant que les juges du fond estiment parfois qu'il n'y a pas d'urgence à suspendre la décision d'installation d'une crèche, dont la dimension de 14 mètres carrés pouvait pourtant prêter à discussion, aucun trouble à l'ordre public ne pouvant être invoqué... (ord. TA Lyon, 17 déc. 2016, à propos de l'installation par Laurent Wauquiez d'une crèche dans le hall de la région Auvergne-Rhône-Alpes). 